\title{
Aplicación de intercambiadores de calor coaxiales en la optimización del cop de sistemas de refrigeración automotriz por compresión del gas R134A.
}

Application of coaxial heat exchangers in the optimization of the cop of automotive cooling systems by compression of the gas R134A.

Paúl Montúfar Paz. ${ }^{1}$, Bolívar Cuaical Angulo. ${ }^{2}$, Ligia Moreno Pinduisaca. ${ }^{3} \&$ Gonzalo Noboa Larrea. ${ }^{4}$

\begin{abstract}
.
DOI: https://doi.org/10.33262/cienciadigital.v2i2.119

The present work has the objective of characterizing several coaxial heat exchanger geometries in an automotive cooling system and its effect on the performance coefficient, using R-134a as refrigerant by means of experimental tests. In the first instance, the experimental tests were carried out with different geometries of coaxial interchanges involved in the high-pressure line between the condenser and the expansion valve, as well as the low pressure line between the evaporator and the compressor, Between the volume of the exchanger and the coefficient of performance by means of a multifactorial analysis. The development of the research entails complying with parameters for the acquisition of experimental data and the development of an adequate experimental design. Statistical regressions were developed with R2 of $86.86 \%$ and a standard deviation of 2.2 for the low-pressure zone, while for the high pressure zone The regression determined a R2 of $95.17 \%$ and a standard deviation of 0.57 . As for the COP and the T variables is statistically significant
\end{abstract}

\footnotetext{
${ }^{1}$ Escuela Superior Politécnica de Chimborazo, Chimborazo Ecuador, pamp6010@ hotmail.com ${ }^{2}$ Escuela Superior Politécnica de Chimborazo, Chimborazo Ecuador, bcuaical@espoch.edu.ec ${ }^{3}$ Escuela Superior Politécnica de Chimborazo, Chimborazo Ecuador, limoreno@espoch.edu.ec ${ }^{4}$ Universidad Estatal de Bolívar, Bolívar, Ecuador, procuraduría@ueb.edu.ec
} 
with a value of R2 of $99.97 \%$, thus helping to design the heat exchangers to decrease fuel consumption in vehicles

Keywords: IHX, COP, R-134 .

\section{Resumen.}

Este trabajo tiene como objeto la caracterización de varias geometrías de intercambiadores de calor coaxiales en un sistema de refrigeración automotriz y su efecto en el coeficiente de desempeño, utilizando como refrigerante el R-134a mediante pruebas experimentales. En primera instancia se realizó las pruebas experimentales con distintas geometrías de intercambiadores coaxiales que intervienen la línea de alta presión entre el condensador y la válvula de expansión, así como la línea de baja presión entre el evaporador y el compresor, determinando una correlación entre el volumen del intercambiador y el coeficiente de desempeño por medio de un análisis multifactorial. El desarrollo de la investigación conlleva cumplir con parámetros para la adquisición de los datos experimentales y el desarrollo de un diseño de experimentos adecuado. Los intercambiadores de calor utilizados fueron de geometría recta con aleación de aluminio 3 003. Se desarrolló regresiones estadísticas con $\mathrm{R}^{2}$ de 86,86 \% y una desviación estándar de 2,2 para la zona de baja presión, mientras que para la zona de alta presión la regresión determino un $\mathrm{R}^{2}$ de 95,17 \% y una desviación estándar de 0,57 . En cuanto al $C O P$ y las variables $T$ es estadísticamente significativa con un valor de $\mathrm{R}^{2}$ de $99,97 \%$, logrando de esta manera ayudar con el diseño de los intercambiadores de calor para disminuir el consumo de combustible en los vehículos.

Palabras Claves: IHX, COP, R-134a .

\section{Introducción.}

El intercambiador de calor interno (IHX) no tiene mucha cabida en el sector automotriz, siendo más ocupado en las plantas de refrigeración con el fin de asegurar una función adecuada y proteger la integridad de los componentes, evitando con esto el ingreso de gas parpadeante a la válvula de expansión y reduciendo con esto el ingreso de líquido a la entrada del compresor. (MACS, 2017)

Mientras menos energía necesite el refrigerante para comprimirse, mayor será el coeficiente de desempeño en el sistema, el coeficiente depende de la combinación de las propiedades térmicas y físicas, el efecto de refrigeración, el volumen específico, el calor específico del líquido, el de evaporación y la relación de compresión. (Goribar, 2003)

En el sector del automóvil, la transición a un refrigerante libre de cloro tuvo lugar a principios de los años 90’s demostrando que el R134a tiene un COP mejor que el $\mathrm{CO}_{2}$ con la disparidad 
COP dependiendo de la velocidad del compresor (capacidad del sistema) y de la temperatura ambiente. Para una velocidad del compresor de 1000 RPM, la COP de CO2 fue menor en un $21 \%$ a $32,2^{\circ} \mathrm{C}$ y en un $34 \%$ a $48,9^{\circ} \mathrm{C}$. A altas velocidades y temperaturas ambiente, la disparidad de COP fue aún mayor. (hidrofluorocarbono, HFC) sustituyó al R12 (CFC). (Brow, Yana, \& Prior, 2002)

Sin embargo, no se presentan investigaciones recientes que ayuden al diseño de un IHX en función de los volúmenes para la fabricación de un intercambiador.

\section{Importancia del problema .}

El consumo de combustibles fósiles ha generado problemas respecto a la contaminación ambiental por lo cual es necesario evaluar alternativas que permitan una utilización más eficiente de los recursos. Al ser los vehículos propulsados por un motor de combustión interna los que encabezan dicho consumo de combustible cualquier medida que logre mermar esta utilización llega a ser de gran importancia.

El uso del aire acondicionado en los automóviles si bien brinda mayor confort a los ocupantes representa también un importante incremento en la demanda energética del trabajo generado por la quema de combustibles, el cual se ha cuantificado entre un 5 y $20 \%$ al consumo normal, el IHX viene a ser una alternativa interesante y de utilidad que permite incrementar el COP del sistema de refrigeración habitual lo cual se traduce en una menor demanda energética de recursos mientras se brinda la misma capacidad de refrigeración de un habitáculo

Para conseguir evaluar el beneficio proporcionado por estos intercambiadores de calor entre las fases de alta y baja presión de un sistema de refrigeración es necesario determinar un modelo estadístico experimental para cuantificar el aumento del COP en función de los volúmenes de transferencia de calor de un intercambiador IHX en un sistema de refrigeración automotriz.

Es necesario además conocer al detalle el comportamiento de cada uno de los componentes que intervienen en el ciclo de refrigeración y del IHX como tal lo cual requiere de caracterizar los componentes y funcionamiento de los sistemas de refrigeración automotriz.

A fin de poder seleccionar el mejor prototipo de intercambiador de calor se seleccionaron varias geometrías que permitan determinar los principales factores de impacto sobre los objetivos del proyecto, para dicho propósito fue necesario analizar diferentes intercambiadores IHX de fácil manufactura mediante pruebas experimentales del sistema de refrigeración en un banco de pruebas. 
Una vez realizadas las pruebas experimentales el determinar la incidencia de la variación de la temperatura en él COP se vuelve de vital importancia para poder conocer los beneficios del sistema.

El sistema de A/C tiene efectos profundos en la economía de combustible del vehículo, la mayoría de sistemas de $\mathrm{A} / \mathrm{C}$ todavía está heurísticamente controlado y opera de manera ineficiente. Un análisis llevado a cabo en Renewable Energy Laboratory mostró que el uso de sistemas es equivalente al 5,5\% del consumo de combustible de vehículos ligeros domésticos. (Quansheng \& Marcello, 2015)

El coeficiente de desempeño de un refrigerante es la medida de su eficiencia en utilizar la energía gastada en el compresor, en relación con la energía absorbida durante la evaporación. Mientras menos energía necesite el refrigerante para comprimirse, mayor será el coeficiente de desempeño en el sistema.

Entonces, el coeficiente de desempeño es la relación entre el efecto de enfriamiento que se realiza en el evaporador $\left(Q_{L}\right)$ y la entrada de trabajo que sucede en el compresor $\left(W_{N E T O}\right)$. (Cengel, 2007)

$$
C O P=\frac{Q_{L}}{W_{\text {neto }}}
$$

\section{Ecuación 1. Coeficiente de desempeño}

Como existe un intercambio de calor durante el ciclo de refrigeración, para esto se debe tomar en cuenta los resultados por cada kg de refrigerante que se tenga disponible en el sistema.

Figura 1. Diagrama presión entalpia con calores aportados y cedidos

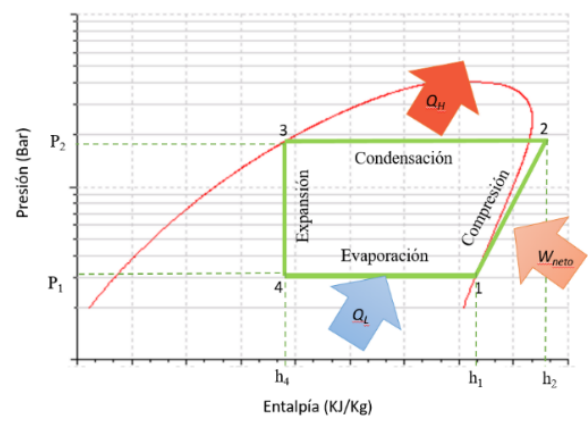

Fuente: Autores, Ecuador, 2017.

Por tanto, como se observa en la figura 1, el trabajo realizado por el compresor en función de las entalpias se representa como la variación de ellas.

$$
\text { Wneto }=h_{2}-h_{1}
$$


De la misma manera el calor absorbido en el evaporador está dado por la variación de las entalpias entre el punto 1 y 4.

$$
Q_{L}=h_{1}-h_{4}
$$

Entonces reemplazando las ecuaciones 1-2 y 1-3 en 1-1 se obtiene:

$$
C O P=\frac{h_{1}-h_{4}}{h_{2}-h_{1}}
$$

Una regla empírica es que el COP mejora entre 2 y 4 por ciento por cada ${ }^{\circ} \mathrm{C}$ que eleva la temperatura de evaporación o que disminuye la temperatura de condensación. (Cengel, 2007)

\section{Intercambiadores de calor.}

Robinson et al. (Douglas \& Eckhard, 1998) presentó una revisión bibliográfica detallada del dióxido de carbono y concluyó que el uso de un intercambiador de calor interno en conjunción con un dispositivo de recuperación de trabajo tiende a reducir el COP del ciclo transcrítico de dióxido de carbono en hasta 8\%. Además, informaron que el uso de un intercambiador de calor interno junto con una válvula de expansión aumenta el COP en hasta un 7\%. (Ciro \& A, 2008)

\section{Intercambiador de calor IHX.}

El Intercambiador de Calor Interno (IHX) es un intercambiador de calor de líquido a vapor, con una cámara interna y una cámara externa. El refrigerante líquido caliente del condensador fluye a través de la cámara interior, y está rodeado por vapor refrigerante fresco que fluye desde el evaporador a través de la cámara exterior.

Después de que el condensador elimina suficiente calor para condensar el refrigerante a un líquido, sigue siendo bastante caliente. El IHX transfiere aún más calor del refrigerante líquido, "sub-enfriando" por debajo de la temperatura de condensación.

Sin embargo, la razón principal de la IHX se utiliza hoy en día es conseguir el uso de menos refrigerante. En el laboratorio, un sistema de A/C lleno de R-134a es aproximadamente un 10\% más eficiente cuando está equipado con un IHX. Eso significa una temperatura más baja en las rejillas de ventilación para la cantidad de energía consumida para hacer funcionar el compresor, reduciendo aún más las emisiones del tubo de escape. (MACS, 2017) 
Figura 2.Intercambiador IHX

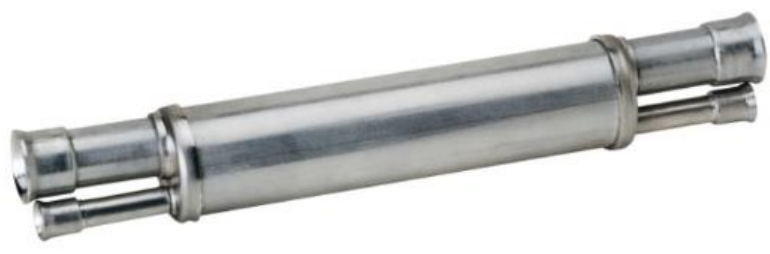

Fuente: : Autores, Ecuador, 2017. (MACS, 2017)

Figura 3. Diagrama presión - entalpía isoterma IHX

Efecto del IHX en el COP

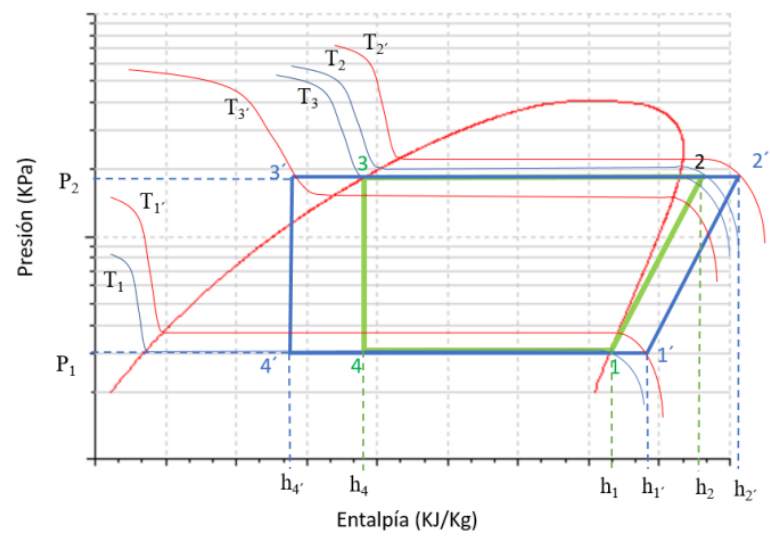

Fuente: Autores, Ecuador, 2017.

En la figura 3 se representa en color verde el ciclo original de funcionamiento del A/C donde sus valores de presión y temperatura para cada punto definen el valor del COP del sistema, este valor del COP puede ser incrementado gracias a la variación de temperaturas de los puntos 1 y 3 , esto se consigue con un intercambiador de calor que se encuentra entre las fases de alta y baja presión pudiéndose diferenciar en la gráfica con color azul que une los puntos del 1'al 4', resultado del cual en los próximos capítulos se cuantificará los valores del COP.

Figura 4. Esquema circuito con IHX 


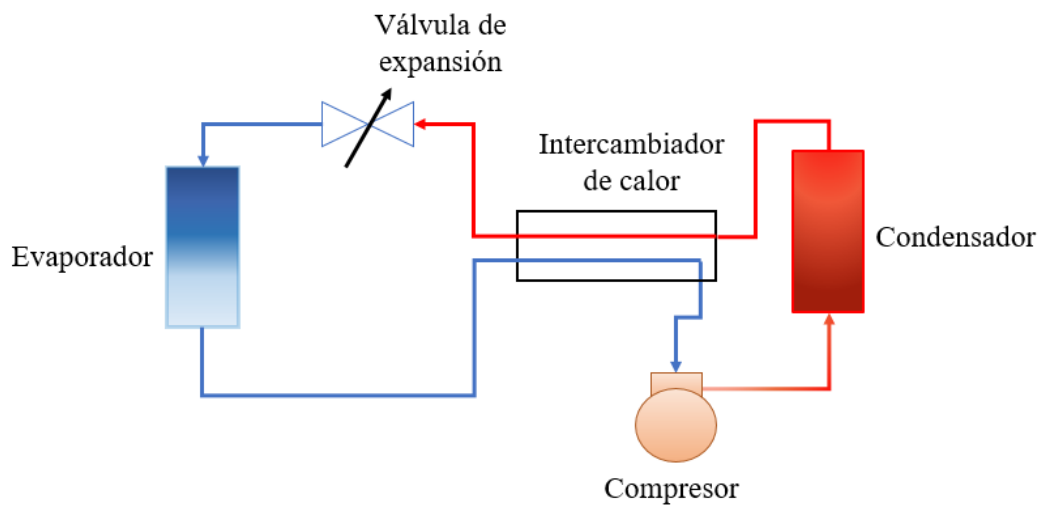

Fuente: Autores, Ecuador, 2017.

\section{Consideración de volumen}

El propósito de considerar los volúmenes del intercambiador para el diseño de los mismos es con el fin de determinar los efectos en los cuales se podrían variar para el diseño del intercambiador.

Se debe tomar en cuenta que existen dos volúmenes, el primero es el volumen interno del intercambiador o el de la cámara interna y el segundo es el que rodea a la cámara interior.

Figura 5. Cámara interna y externa del intercambiador.

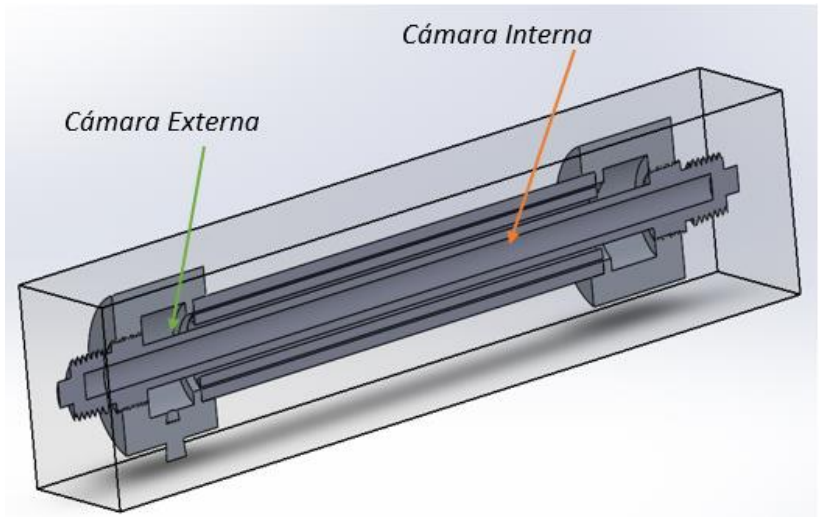

Fuente: Autores, Ecuador, 2017.

\section{Diseño de experimentos}

El diseño de experimentos (DOE) es una técnica estadística que se basa en organizar y diseñar una serie de experimentos de forma que con el mínimo número de pruebas se consiga extraer información útil para obtener conclusiones que permitan optimizar la configuración de un proceso o producto. (Bernal, 2017)

Figura 6. Diseño Factorial DOE 


\section{Diseño factorial de múltiples niveles}

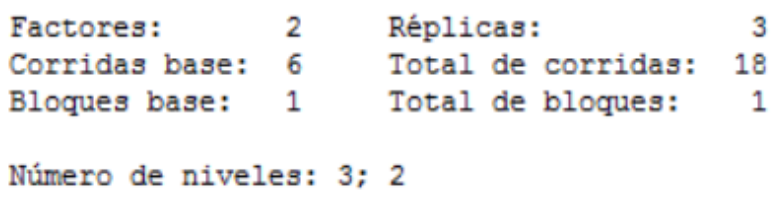

Fuente: Autores, Ecuador, 2017.

\section{Metodología.}

En los experimentos se realizarán de acuerdo al diseño experimental dieciocho corridas con el sistema de aire acondicionado incorporando los intercambiadores de calor en velocidad uno y en velocidad dos, con el fin de recopilar los datos de temperatura y presión en cada uno de los puntos críticos del sistema, los mismos que serán tomados en las mismas condiciones que en las corridas del sistema en condiciones normales.

\section{Banco de pruebas.}

Las pruebas experimentales para la caracterización del ciclo de refrigeración se las realiza en el laboratorio de aires acondicionados de la Escuela superior politécnica de Chimborazo, mediante el uso del banco de pruebas de aires acondicionados automotrices, el mismo que brinda la posibilidad de tomar las lectura tanto de presiones como de temperaturas a las cuales funciona un sistema de aires acondicionado automotriz, adicional a esto se puede realizar pruebas continuas y recopilar estos parámetros en tiempo real por medio de una tarjeta de adquisición de datos.

Figura 7. Banco de aires acondicionados automotrices.

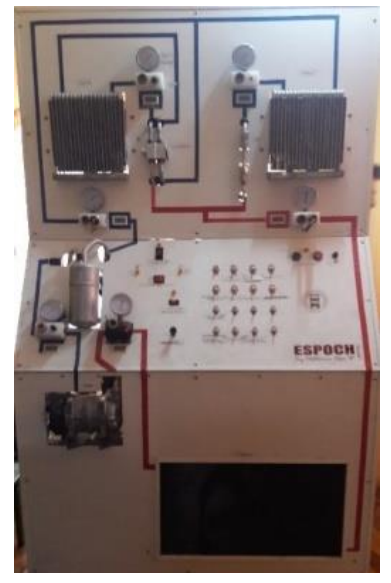

Fuente: Autores, Ecuador, 2017. 
Sensor de presión (circuito de baja presión)

Los sensores determinados para la recopilación de datos del circuito de baja presión es el transmisor de presión de tipo HR-PTM300 y es uno de los más aplicados para la medición de aire y líquido.

Figura 8.Sensor transmisor de presión HR-PTM300.

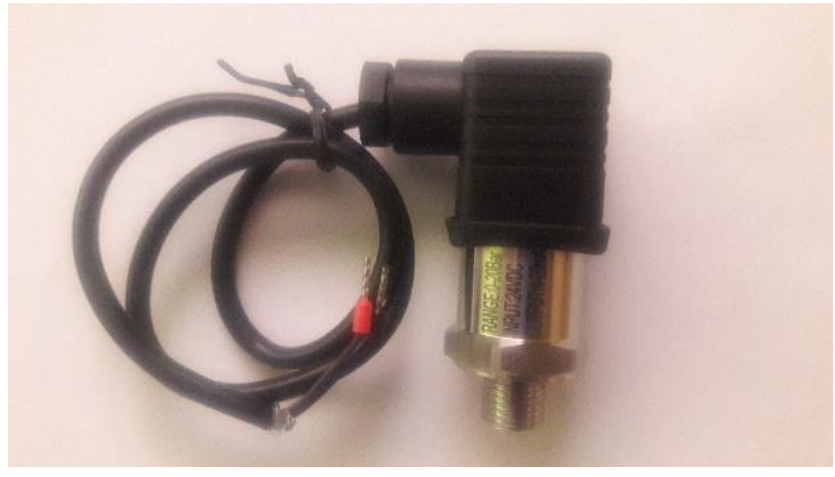

Fuente: Autores, Ecuador, 2017.

\section{Sensor de presión (circuito de alta presión).}

Los sensores determinados para la recopilación de datos del circuito de alta presión es el sensor de presión de tipo HK-1100C y es uno de los más aplicados para la medición de en la industria.

Figura 9.Sensor transmisor de presión HK-1100C.

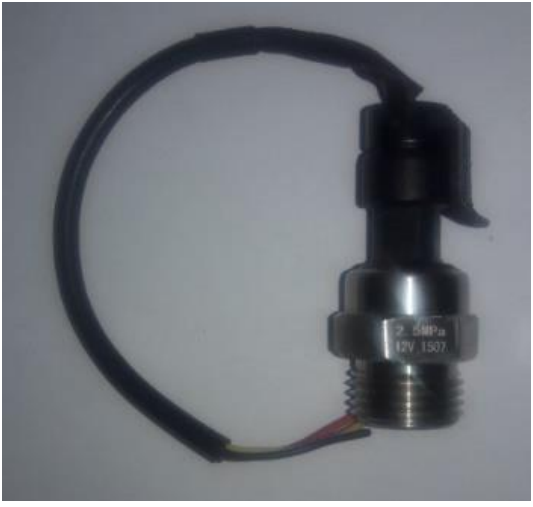

Fuente: Autores, Ecuador, 2017.

\section{Sensor de temperatura.}

Los sensores determinados para la recopilación de datos del circuito de alta y baja presión son los sensores de NTC-10k, el mismo que es un termistor que al aumentar la temperatura, razón por la que también aumenta sus componentes internos, motivo por el cual su resistencia es menor. 
Figura 10. Sensor de temperatura NTC-10K.

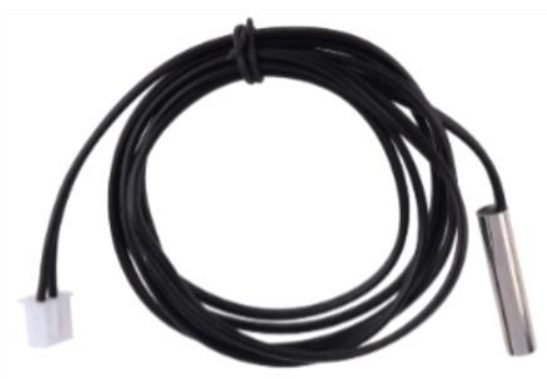

Fuente: Autores, Ecuador, 2017.

\section{Compresor.}

El compresor que se utilizara en el banco de pruebas es un compresor de carrera variable y funciona con dos velocidades a $1750 \mathrm{rpm}$ y $1805 \mathrm{rpm}$.

\section{Caracterización del ciclo de refrigeración.}

Para la caracterización del sistema normal del aire acondicionado se establecen los valores tanto de presión como de temperatura medidos a las salidas del compresor, condensador y evaporador, para posteriormente realizarla incorporando ya el intercambiador de calor, con valores medidos a las entradas y salidas del intercambiador y de esta manera lograr predecir los rangos de operación y verificar el incremento del COP.

Resultados.

Como se explicó anteriormente la variación de la temperatura tanto en la entrada del compresor como a la entrada de la válvula de expansión determinan el aumento del coeficiente de desempeño, los mismos que se valoraron para determinar su comportamiento.

Variación de temperatura en la entrada del compresor.

Una vez determinados los valores tanto de temperatura como de presión con el ciclo normal de funcionamiento del sistema de aire acondicionado, se procede a determinar el comportamiento del COP en cuanto al aumento de temperatura en la entrada del compresor. 
Figura 11. Variación COP vs. T entrada del compresor.

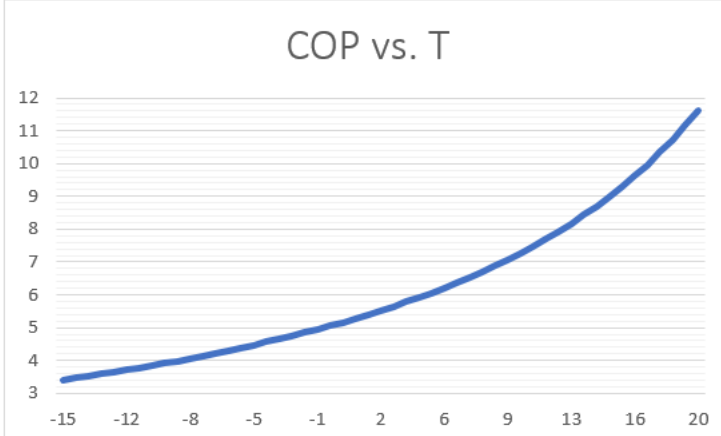

Fuente: Autores, Ecuador, 2017.

\section{Variación de temperatura en la entrada de la válvula de expansión.}

A continuación, se procede a determinar el comportamiento del COP en cuanto a la disminución de temperatura en la entrada de la válvula de expansión.

Figura 12. Variación COP vs. T entrada válvula de expansión.

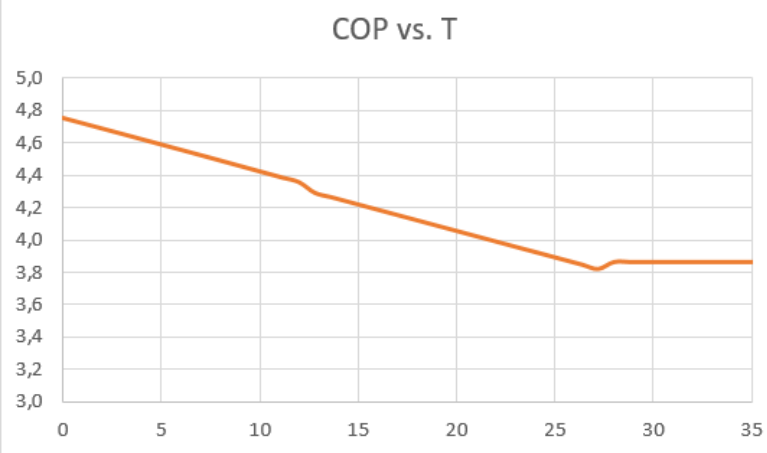

Fuente: Autores, Ecuador, 2017.

\section{Discusión.}

Con los datos obtenidos y utilizando el software Minitab se procede a realizar una regresión seleccionando los mejores subconjuntos para determinar los valores de la variación de temperatura con respecto a los volúmenes de la cámara interna como de la externa. 
Figura 13. Predictor variación de temperatura tramo de baja presión.

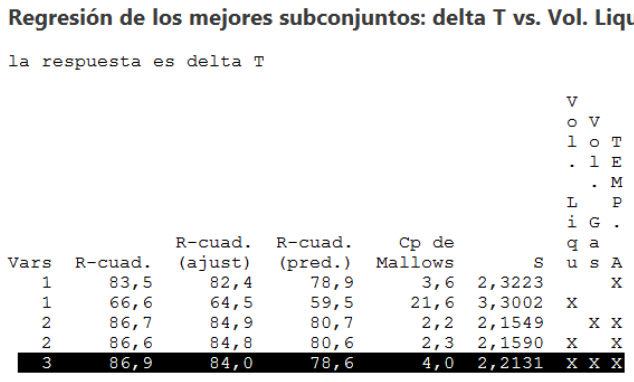

Fuente: Autores, Ecuador, 2017.

Por medio del software se logra determinar que $\mathrm{R}^{2}$ es del $86,9 \%$, lo que nos indica que las variables seleccionadas son las correctas ya que mientras más alto sea este valor, mejor será el ajuste del modelo a los datos.

Figura 14. Probabilidad normal regresión $\Delta T$ tramo de baja presión.

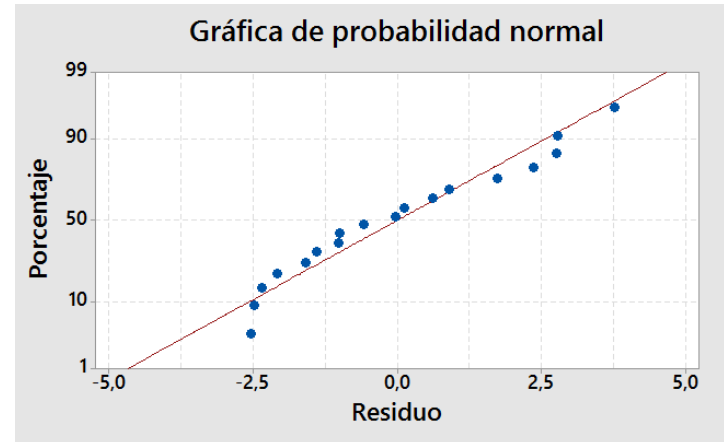

Fuente: Autores, Ecuador, 2017.

Determinada la regresión se resume el modelo con un $\mathrm{R}^{2}$ de $86,86 \%$ y una desviación estándar de 2,213 y obteniendo una ecuación justificada experimentalmente.

$$
\Delta T=14,7+0,00448 V_{L}-0,00161 V_{G}-0,63 T_{A}
$$

El decremento de la temperatura en el punto crítico tres antes de la válvula de expansión es importante para el incremento del COP como se pudo determinar anteriormente, por lo tanto, es necesario dar un tratamiento estadístico al modelo experimental en relación de la variación de temperatura que se provoca en el intercambiador de acuerdo a las tres geometrías evaluadas. 
Figura 15. Predictor variación de temperatura tramo de alta presión.

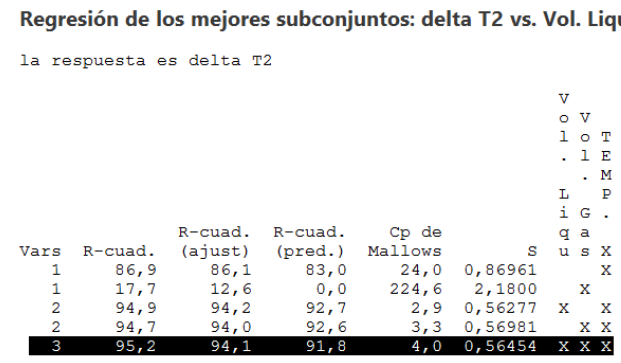

Fuente: Autores, Ecuador, 2017.

Por medio del software se logra determinar que $\mathrm{R}^{2}$ es del 95,2\%, lo que nos indica que las variables seleccionadas son las correctas ya que mientras más alto sea este valor, mejor será el ajuste del modelo a los datos.

Figura 16. Probabilidad normal regresión $\Delta T$ tramo de baja presión.

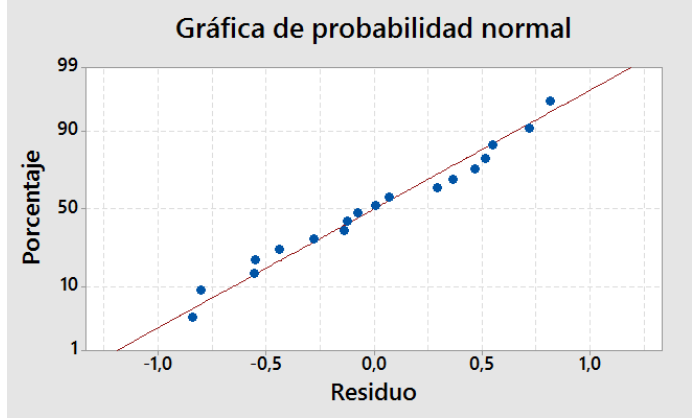

Fuente: Autores, Ecuador, 2017.

Determinada la regresión se resume el modelo con un $\mathrm{R}^{2}$ de 95,17 \% y una desviación estándar de 0,565 y obteniendo una ecuación justificada experimentalmente.

$$
\Delta T_{2}=-16,75+0,000670 V_{L}-0,000207 V_{G}+0,7089 T_{A}
$$

Determinadas las ecuaciones que nos ayudan con la determinación de los valores de la variación de temperatura en los dos tramos del circuito se procede a realizar una regresión estadística para determinar el efecto de la variación de temperatura en el COP, obteniendo los siguientes resultados.

$$
C O P=4,114+0,1912 T_{A}+0,0312 T_{A}^{2}+0,0101 T_{X}^{2}+0,2152 T_{A} x T_{X}
$$


Figura 17. COP Vs $T_{A}$ y $T_{X}$.

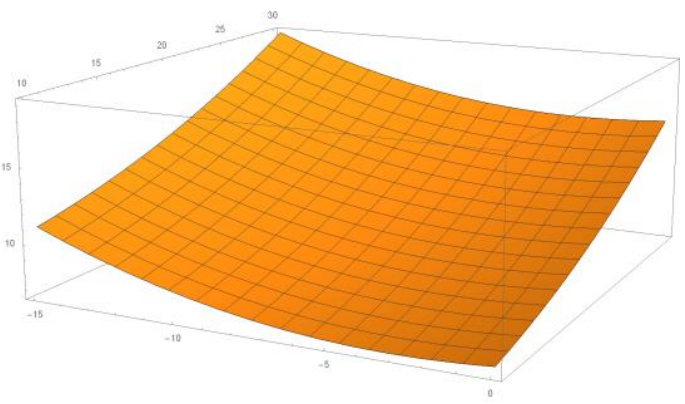

Fuente: Autores, Ecuador, 2017.

\section{Conclusiones.}

- La implementación de intercambiadores de calor coaxiales en los sistemas de refrigeración automotriz proporcione ventajas de eficiencia en el consumo para ciertas zonas de operación, estas ventajas están en el orden del $4 \%$ respecto a sistemas de aire acondicionado estándar, por tal motivo esta investigación brinda ventajas ambientales y económicas que permitirán un punto de partida dentro de la optimización de sistemas de refrigeración por compresión del R134A.

- La forma de los intercambiadores de calor es un factor importante al momento de optimizar espacios en el habitáculo de motor en vista de formas curvas ofrecen mayor incremento de COP por metro cuadrado de material con que manufacturo los intercambiadores.

- De acuerdo a los resultados se logró obtener una estimación precisa de la relación, manteniendo unos pocos datos con residuos grandes, pero estos valores fuera de lo normal no representan un problema para la normalidad del modelo.

- Se determinó una correlación entre los volúmenes de la cámara interna como la externa en relación al COP, estableciendo parámetros importantes para el diseño de intercambiadores de calor para estos sistemas.

- La relación entre el $C O P$ y las variables $T$ es estadísticamente significativa con un valor de $\mathrm{R}^{2}$ de $99,97 \%$, por lo que esta ecuación se puede utilizar para predecir el COP para valores específicos de las variables $\mathrm{T}$, o para encontrar la configuración de las variables $\mathrm{T}$ que corresponda a un valor o rango de valores deseado para el COP. 


\section{Referencias bibliográficas.}

International Journal of Refrigeration, Brow, J. S., Yana, S. F., \& Prior, D. A. (2002). Comparitive analysis of an automotive air conditioning systems operating with $\mathrm{CO} 2$ and R134a. International Journal of Refrigeration, 19-32.

International Journal of Refrigeration Mota-Babiloni, A., Navarro-Esbrí, J., BarragánCervera, Á., Molés, F., \& Peris, B. (2014). Analysis based on EU Regulation No $517 / 2014$ of new HFC/HFO mixtures as alternatives of high GWP refrigerants in refrigeration and HVAC systems. International Journal of Refrigeration, 21-31.

Mobility Air Conditionig Society. MACS. (06 de Enero de 2017). Mobility Air Conditionig Society. Obtenido de https://macsworldwide.wordpress.com/2013/08/02/what-is-aninternal-heat-exchanger/

pdcahome.B ernal, J. J. (17 de 04 de 2017). Obtenido de pdcahome: http://www.pdcahome.com/2117/diseno-de-experimentos-para-que-sirve-y-comorealizarlo/

Termodinámica. Cengel. (2007). Termodinámica. Mc Graw Hill. 
Para citar el artículo indexado.

Montúfar P, Bolívar A., Moreno L. \& Noboa G. (2018). Aplicación de intercambiadores de calor coaxiales en la optimización del cop de sistemas de refrigeración automotriz por compresión del gas r134a. Revista electrónica Ciencia Digital 2(2), 561-577. Recuperado desde:

http://cienciadigital.org/revistacienciadigital2/index.php/CienciaDigital/article/view/119/11 $\underline{0}$

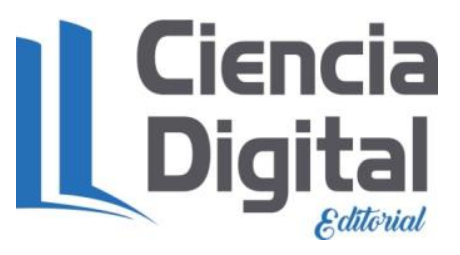

El artículo que se publica es de exclusiva responsabilidad de los autores y no necesariamente reflejan el pensamiento de la Revista Ciencia Digital.

El articulo queda en propiedad de la revista y, por tanto, su publicación parcial y/o total en otro medio tiene que ser autorizado por el director de la Revista Ciencia Digital.
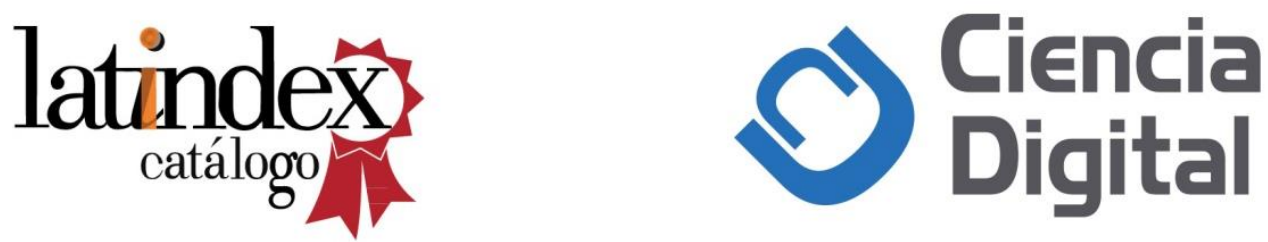